\title{
Advancing the CERN proton synchrotron multiturn extraction towards the high-intensity proton beams frontier
}

\author{
A. Huschauer, H. Bartosik, S. Cettour Cave, M. Coly, D. Cotte, H. Damerau, \\ G. P. Di Giovanni, S. Gilardoni, M. Giovannozzi $\odot{ }^{*}$ V. Kain, E. Koukovini-Platia, \\ B. Mikulec, G. Sterbini, and F. Tecker \\ Beams Department, CERN, 1211 Geneva 23, Switzerland
}

(Received 20 December 2018; published 24 October 2019; corrected 30 October 2019)

\begin{abstract}
Complementary to the physics research at the LHC, several fixed-target facilities receive beams from the LHC injector complex. To serve the fixed-target physics programme at the super proton synchrotron, high-intensity proton beams from the proton synchrotron are extracted using the multiturn extraction technique based on trapping parts of the beam in stable resonance islands. Considering the number of protons requested by future experimental fixed-target facilities, such as the proposed search for hidden particles experiment, the currently delivered beam intensities are insufficient. Experimental studies were conducted to optimize the multiturn extraction technique, pushing its capabilities in the domain of highintensity proton beams, and their results are presented in this paper. The success of these studies led to the decision to discontinue the former continuous transfer and remove the related hardware from the accelerator. Therefore, the multiturn extraction becomes standard operational practice for delivering proton beams for the fixed-target physics programme at the CERN super proton synchrotron.
\end{abstract}

DOI: 10.1103/PhysRevAccelBeams.22.104002

\section{INTRODUCTION}

Since September 2015, the special beam extracted from the CERN proton synchrotron (PS) for the super proton synchrotron (SPS) fixed-target physics program has been generated using the so-called multiturn extraction (MTE) technique (see [1-7] for more detail). This peculiar extraction technique has superseded the continuous transfer (CT) process, proposed in 1973 [8], which occurs over five turns at $14 \mathrm{GeV} / c$ to optimize the duty cycle by filling the SPS with only two subsequent extractions from the PS. The MTE technique was developed to overcome the downside of the CT extraction, namely significant beam loss occurring at multiple locations around the ring [9], leading to high-radiation dose to personnel during accelerator maintenance and repair, long cool-down times, as well as to shorter lifetime of accelerator components.

The essence of MTE is the beam splitting process [1,4-6], performed by crossing a stable, fourth-order resonance in the horizontal plane. Two distinct structures of different length are generated: (i) the beam left around

\footnotetext{
Corresponding author. massimo.giovannozzi@cern.ch

Published by the American Physical Society under the terms of the Creative Commons Attribution 4.0 International license. Further distribution of this work must maintain attribution to the author(s) and the published article's title, journal citation, and DOI.
}

the origin, which has a length of one PS turn and is referred to as the core and (ii) the beam trapped in the resonance islands, with a length of four PS turns. These two structures are extracted sequentially, first the islands and then the core. A slow bump approaches them to the magnetic septum and three kickers create a fast bump, lasting five turns, around the extraction septum to deflect the islands out of the PS ring. The core is extracted by imparting an additional deflection by means of two more kickers. In this respect, two separate stages are needed for MTE, i.e., beam splitting (to generate the suitable structures to be extracted over five turns) and beam extraction proper.

Figure 1 shows a sketch of the PS ring with the main nonlinear magnets required for MTE, namely sextupoles and octupoles. While the sextupoles and the close-by octupoles are located in areas with maximum horizontal and minimum vertical $\beta$-functions to enhance their effect, the other distributed octupoles are located in areas with maximum vertical and minimum horizontal $\beta$-functions and are used to minimize the nonlinear coupling between the two transverse planes [4-6]. For the sake of clarity, a summary of the main parameters of the PS and SPS rings is listed in Tables III and IV, respectively, in the Appendix (from [5]).

The PS cycle for SPS filling is shown in Fig. 2 (upper) together with the evolution of the strengths of the nonlinear magnets used to perform beam trapping and splitting (lower). 


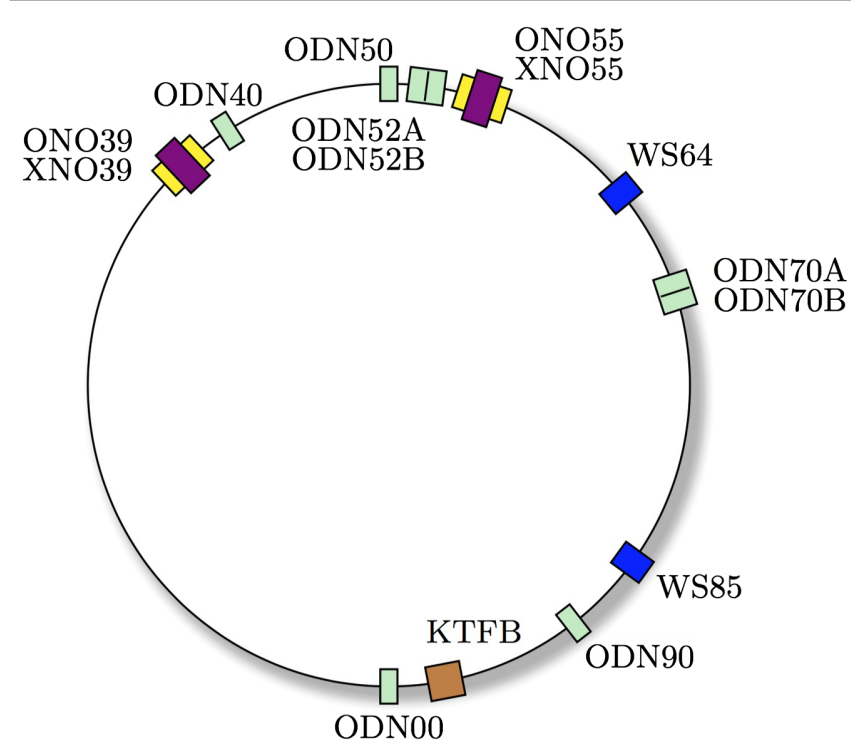

FIG. 1. Sketch of the PS ring with the key elements of MTE, i.e., sextupole (called "X") and octupole (called "O") magnets. "ON" octupoles are used to generate and manipulate the islands, whereas "OD" octupoles are used to control the nonlinear transverse coupling. The positions of the kicker of the transverse feedback (KTFB) and of the vertical wire scanners (WS) are indicated as well.

The efficiency of the transverse splitting is defined as

$$
\eta_{\mathrm{MTE}}=\frac{\left\langle I_{\text {Island }}\right\rangle}{I_{\text {Total }}}
$$
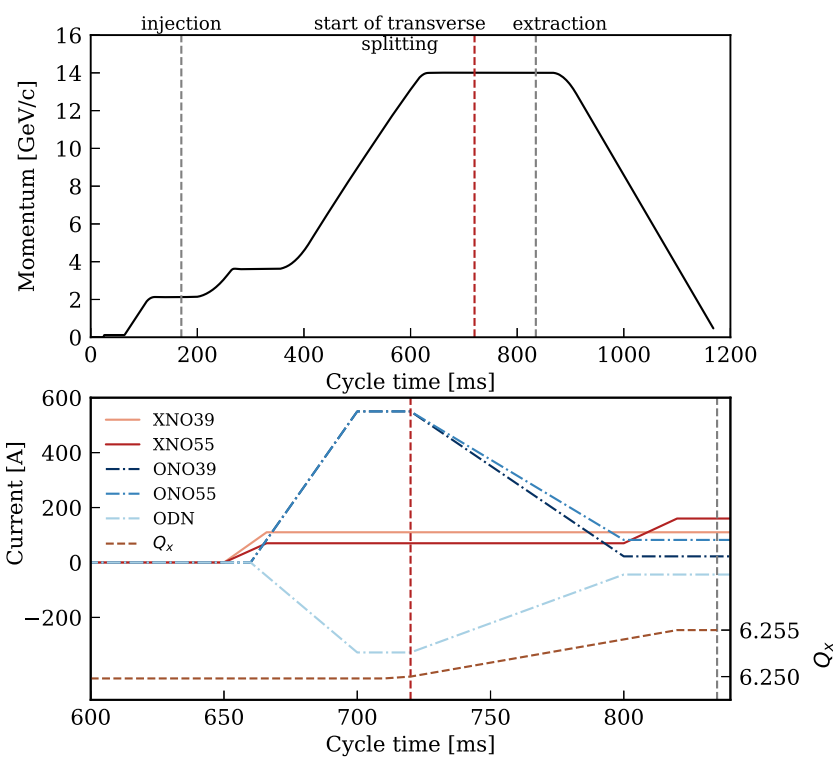

FIG. 2. Upper: Sketch of the PS magnetic cycle with the main events. Lower: Evolution of the strengths of the main MTE elements. The red vertical dashed line indicates the moment of resonance crossing and the grey one the extraction. where $\left\langle I_{\text {Island }}\right\rangle$ and $I_{\text {Total }}$ stand for the average intensity in each island and the total beam intensity, respectively. The nominal efficiency is 0.20 , corresponding to an equal beam sharing between islands and core. This figure of merit is derived from the signal of the beam intensity measured in the transfer line connecting the PS and the SPS.

To satisfy the requests of the SPS fixed-target experiments, the typical intensity per PS extraction was in the range of $N_{\mathrm{p}}=1.5-2 \times 10^{13}$ protons (p) in the years 2015-17. Note that during the CERN Neutrino to Gran Sasso [10] run, the typical proton intensity extracted from the PS was $N_{\mathrm{p}} \sim 2.6 \times 10^{13} \mathrm{p}$.

According to future proposals, like the search for hidden particles (SHiP) experiment [11], much higher intensity, reaching up to $2.4-2.5 \times 10^{13}$ protons per PS extraction, might be required. Therefore, in light of these potential needs, an extended experimental campaign was carried out in 2017 to assess in great detail the MTE performance for high-intensity beams. Intensity-dependent effects had been observed with MTE already during its infancy [12], while their theoretical explanation, based on the analysis of indirect space charge effects, has been provided only recently [13]. Hence, the results of these high-intensity tests are an important milestone as, for the first time ever, they imply the capability of mastering complicated phenomena stemming from the interplay between external nonlinearities and space-charge (direct or indirect) effects. Future research activities aim at simulating these effects to predict the MTE behavior in this new regime. Another aspect making these experimental studies so relevant is that their successful outcome was considered an essential step in the formal process of declaring MTE a suitable and definitive operational replacement of CT.

This paper is based upon [14] and extends the findings presented therein. In Sec. II, a number of detailed parameter scans are presented to assess the performance of MTE as function of the transverse excitation applied during the splitting and of the strength of the nonlinear magnetic elements. These studies were a crucial prerequisite to start beam tests at higher intensity. In Sec. III the detailed discussion of the high-intensity tests is presented, including the various steps undertaken in the whole accelerator chain starting from the PS Booster (PSB) to the PS and the SPS. Finally, concluding remarks are presented in Sec. IV.

\section{PREPARATORY STUDIES: OPTIMIZATION OF TRANSVERSE EXCITATION, NONLINEAR MAGNETS, AND TRANSVERSE EMITTANCES}

A non-negligible boost to $\eta_{\text {MTE }}$ is provided by the use of a horizontal dipolar excitation during the resonancecrossing process. Such an excitation is imparted by the transverse feedback (TFB) system used in open loop and its impact was analysed in detail in the past [6]. The use of 
such a transverse dipolar excitation is essential to achieve the nominal value of $\eta_{\mathrm{MTE}}$.

The transverse emittances of the operational beams are measured on a regular basis in the SPS. Occasionally, these measurements revealed a very large horizontal emittance of the core, accompanied by an increase in beam loss. Given the important role of the dipolar excitation during the splitting process, the core emittance growth was expected to be a negative side effect nonoptimal TFB settings. This possibility was verified by a detailed measurement campaign, where $\eta_{\mathrm{MTE}}$ and the horizontal emittance growth of the core were measured as a function of the excitation frequency [14]. The measurements clearly revealed that the transverse feedback can indeed be tuned and optimized to maximize its beneficial effect, while keeping the undesired impact on the core emittance under control.

The operational settings of the nonlinear magnets shown in Fig. 2 (lower) were defined to maximize $\eta_{\mathrm{MTE}}$ (by means of ONO39 and ONO55), to minimize the nonlinear coupling between the two transverse planes (by means of the ODN family), and to reduce emittance dilution and extraction losses during the change of the islands' phase prior to extraction (by means of the XNO55 circuit) [6].

Some of the features of the time variation of the strengths of the sextupole and octupole circuits were revised in view of the high-intensity tests to further optimize the initial MTE configuration.

The results presented in Figs. 3-6 are based on random variations of the independent parameter while measuring the dependent parameter. Each plot is the result of hundreds of subsequent measurements, where each one was performed on a different cycle and the reconstructed distribution of the measured points is shown. This has the advantage of providing a more detailed view of the functional relationship between the various parameters. During these measurements, the amplitude of the transverse excitation was reduced in order to probe the direct impact of the strengths of the nonlinear magnets on the MTE efficiency or the losses at extraction.

At first, the settings of the octupole circuits were probed to assess whether their maximum strength or the slope from the maximum value at resonance crossing to the final one before extraction were optimal. The results of these scans in terms of distribution of measured $\eta_{\mathrm{MTE}}$ are shown in Fig. 3. A strong dependence on the value of the maximum strength is clearly observed, while a mild increase of the MTE efficiency is measured when the slope is reduced, i.e., the time variation and therefore the adiabaticity of the process is increased.

The impact of the maximum strength of the ODN magnets, which are meant to control the nonlinear coupling between the two transverse planes, was probed too, and the results are shown in Fig. 4. The measurements show that $\eta_{\text {MTE }}$ is essentially independent of the maximum amplitude programmed in the current function of the ODN magnets.
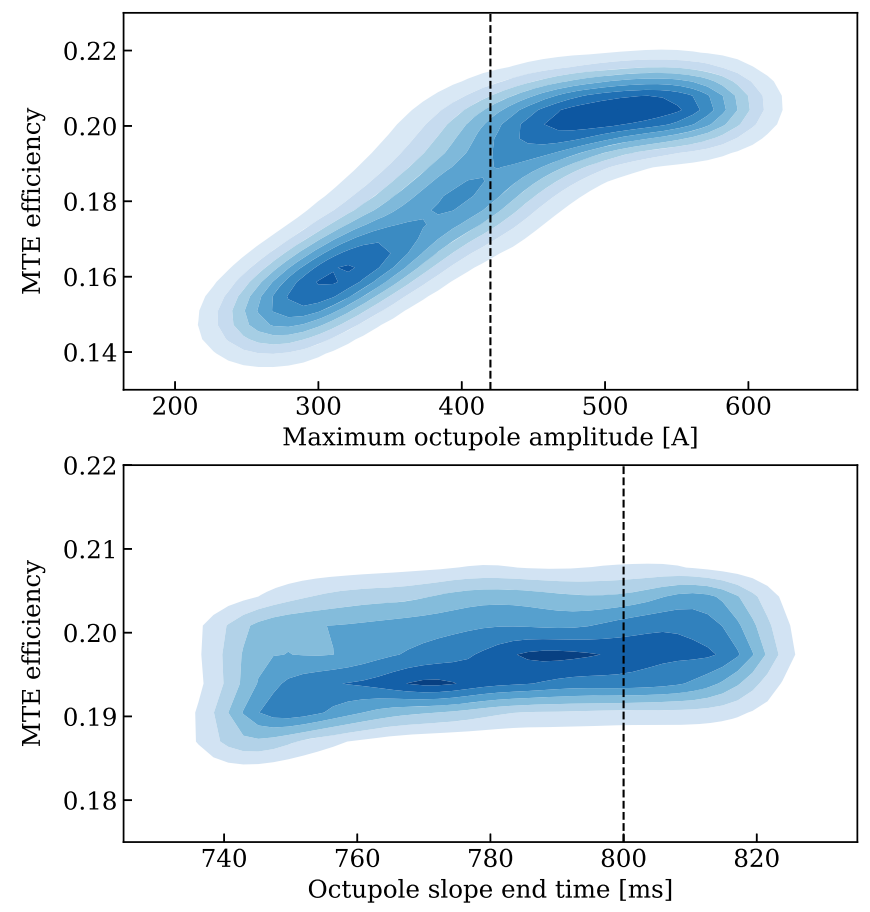

FIG. 3. Distribution of $\eta_{\mathrm{MTE}}$ as a function of the maximum strength of octupoles ONO39 and ONO55 (upper) and of their slope (lower). A strong dependence of $\eta_{\mathrm{MTE}}$ on the strength is visible, while the slope is affecting it only mildly. The vertical dashed lines represent the values of the operational settings.
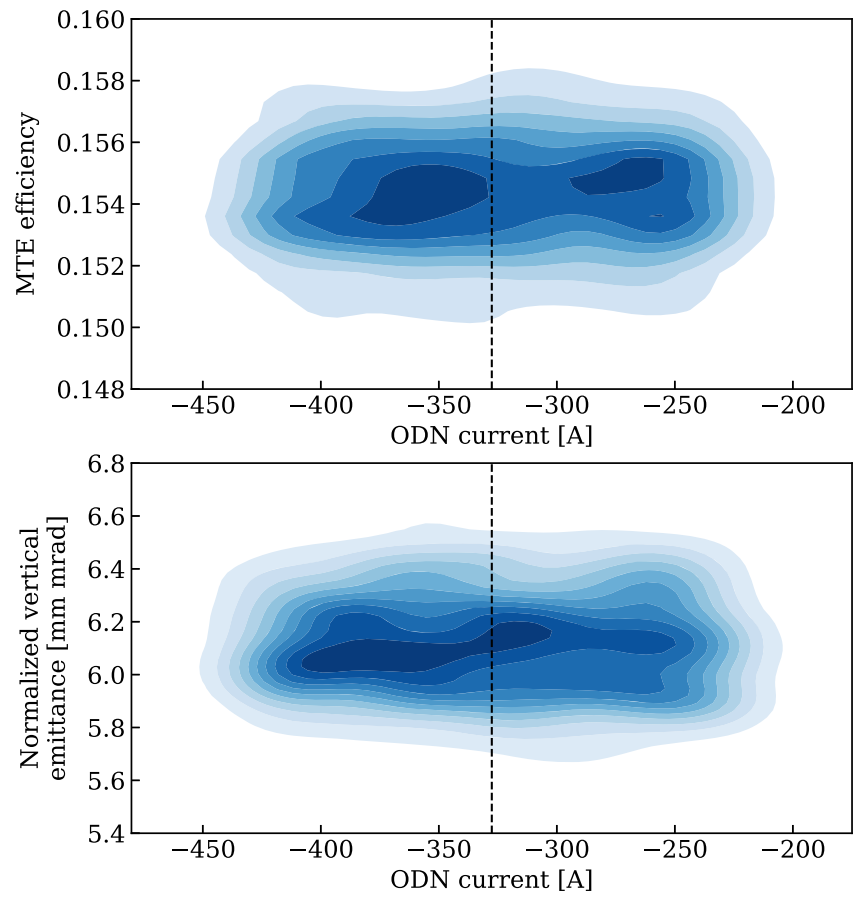

FIG. 4. Distribution of $\eta_{\mathrm{MTE}}$ (upper) and vertical emittance (lower) as a function of the maximum strength of octupoles ODN. No dependence of both parameters on the strength is visible in the explored regime. The vertical dashed lines represent the value of the operational current. 

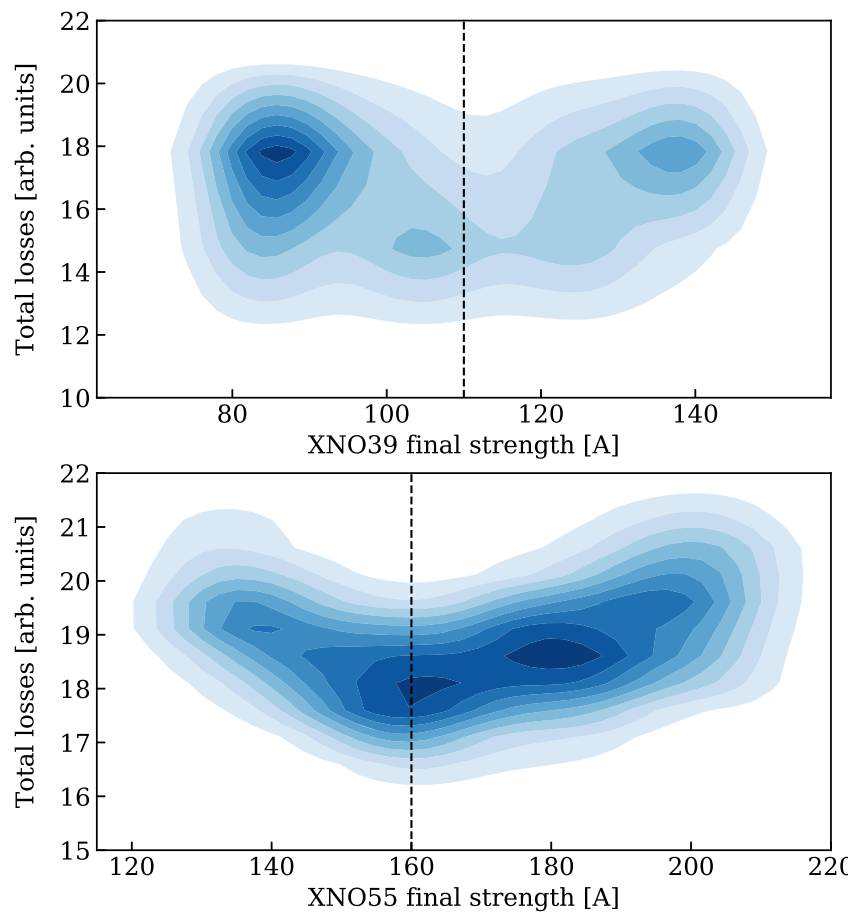

FIG. 5. Distribution of extraction losses as a function of the final strength of the sextupoles XNO39 (upper) and XNO55 (lower) during the separation of the islands and the change of their phase. A clear dependence is visible for XNO55, while almost no dependence is observed for XNO39. The vertical dashed lines represent the values of the operational currents.

Furthermore, the vertical emittance appears to be independent of the ODN current within the probed regime. These results have to be compared to previous experimental studies, in which the complete absence of the ODN strength led to a significant decrease of $\eta_{\mathrm{MTE}}$ [3].

As a final test, the strength of the sextupoles XNO39 and XNO55 was varied, and their impact on the extraction losses, as measured by means of beam loss monitors (BLMs), is reported in Fig. 5. The strength varied corresponds to the final stage of the resonance crossing process, i.e., when the islands are transported toward higher amplitude and their phase is changed in order to prepare for the extraction. Close to no dependence is observed for the losses as a function of the strength of XNO39, and the operational setting corresponds to the optimal value. On the other hand, the losses show a clear dependence on the current of XNO55, with a minimum occurring again at the operational value of the current.

The constraints on the transverse emittances for the MTE fixed-target beams are three-fold: (i) the horizontal emittance received by the PS should be large to increase $\eta_{\mathrm{MTE}}$, (ii) the vertical emittance should be as small as possible to overcome the acceptance issues in the SPS, and (iii) extraction losses at the PSB should be kept low.

The detailed discussions of the PSB studies to optimize the transverse emittances of the MTE beam can be found

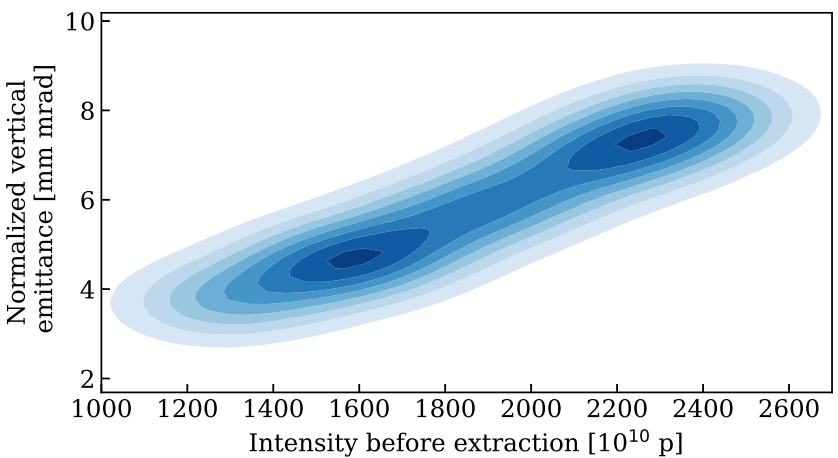

FIG. 6. Distribution of the normalized vertical emittance measured with WS85 before PS extraction as a function of the total beam intensity after the optimization carried out at the PSB. A linear dependence between emittance and intensity is clearly visible.

in [14]. Here, Fig. 6 is reported to account for the dependence of the vertical normalized emittance on intensity, measured with a wire scanner (WS) just prior to PS extraction after the special optimisation carried out at the PSB. A linear dependence is clearly visible, indicating constant beam brightness. The two peaks of the density distribution represent the typical values of the vertical emittance of the operational beam and of the special beam used during the high-intensity tests reported in the following section.

\section{HIGH-INTENSITY TESTS}

Based on the results presented in the previous section and the subsequent optimisation of the settings, the PS cycle became ready for high-intensity beam production. The SPS settings on the other hand had to be extensively retuned to enable the tests with the high-intensity beam. Due to the limited available time the setup could only be completed partially and will be further addressed in future studies.

In the following the PS and SPS performance with highintensity MTE beams will be reported.

\section{A. PS results}

During the high-intensity tests four aspects were scrutinized at the PS: (i) the dependence of the vertical emittance as a function of intensity and along the PS magnetic cycle, (ii) the dependence of $\eta_{\mathrm{MTE}}$ on intensity, (iii) the dependence of extraction losses on intensity, and (iv) the beam behavior in the longitudinal plane during the debunching applied after splitting and prior to beam extraction.

The last point will not be discussed in this paper as no particular issue was observed and the beam could be kept stable in the longitudinal plane during the debunching even at the highest intensities.

The evolution of the vertical beam emittance measured in the PS during the 2017 run is shown in Fig. 7 as a function 

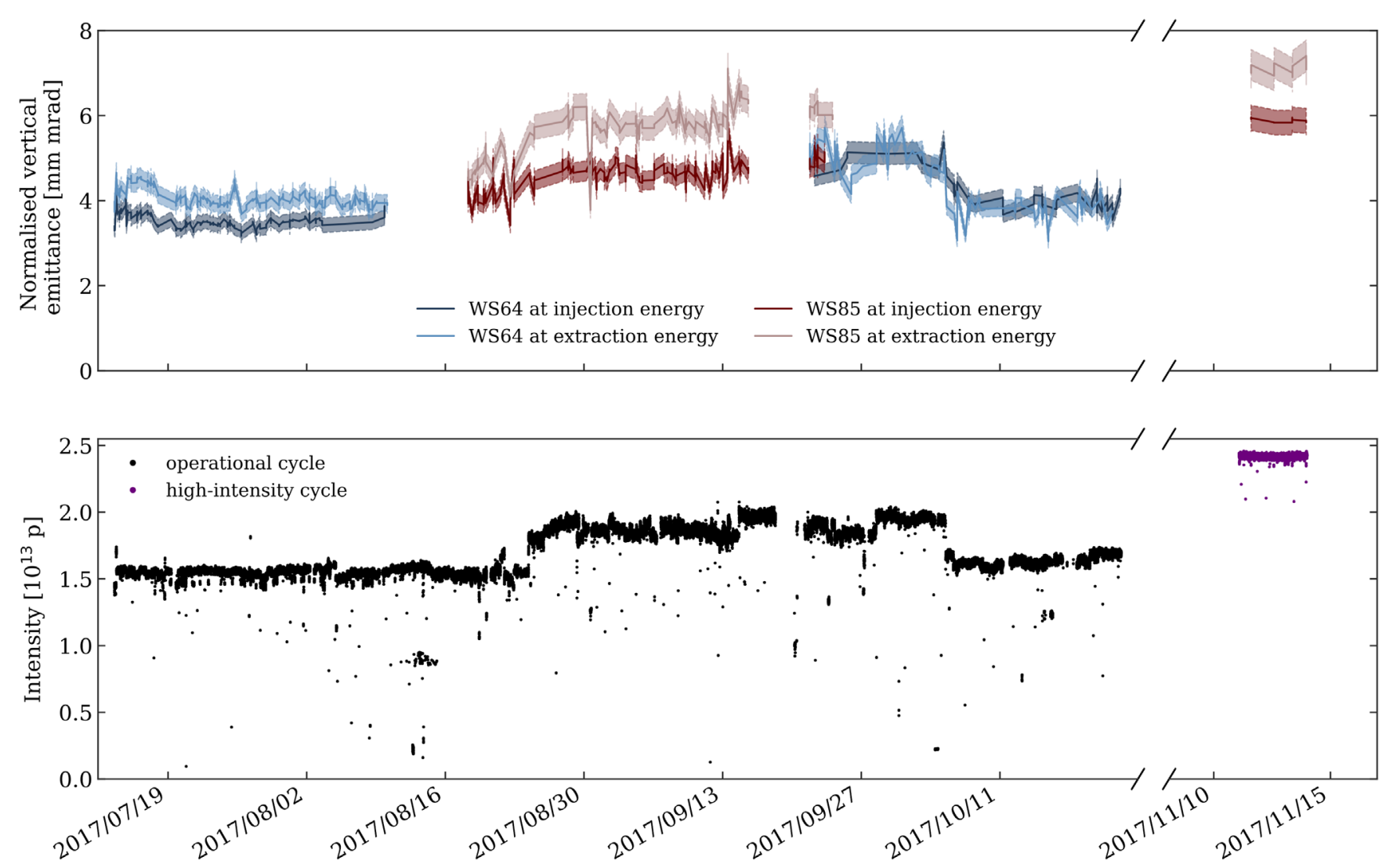

FIG. 7. Evolution of the vertical beam emittance measured at PS injection (190 ms) and just before transverse splitting (700 ms) for the two wire scanners (upper). The coloured bands around the measured emittance values indicate the error bars, which account for the goodness of the Gaussian fit and the beta-beating at the WS locations. The lower plot shows the circulating beam intensity before extraction over time. Data is shown for the operational period (until end of October 2017) and for the high-intensity tests in November 2017. The different steps in intensity for the operational beam indicate different requirements for the fixed-target physics run.

of time together with the circulating beam intensity. The high-intensity tests were carried out in November 2017 and vertical emittance and intensity data are therefore presented at the right end of Fig. 7. The intensity variations that occurred in August and October 2017 reflect the different needs for the fixed-target physics run.

The vertical emittance was measured at two different instances along the PS cycle, i.e., just after injection and just prior to crossing the fourth-order resonance. The emittance value is obtained by fitting the transverse profiles measured with the wire scanners in sections 64 or 85 of the PS ring. The use of both devices was necessary, as the wire of scanner 64 broke in August 2017 and a repair could only be performed during a technical stop in September 2017.

The raw data were cleaned by removing any baseline variation and then fitted with a Gaussian. After this procedure, only the profiles satisfying the condition $R^{2}>0.995$ were retained and used for the following analysis. The error bar associated to the computed emittance value is based on the goodness of the Gaussian fit and includes an error of $5 \%$ on the $\beta_{y}$-value at the location of the wire scanner, which represents the typical value of betabeating according to a detailed measurement campaign reported in [15]. In Fig. 7 (upper) the lines represent the computed emittance value, whereas the lighter color bands around them the associated errors.

The difference between the two bands indicates any source of emittance growth occurring in the PS. It is clearly visible that the measurements performed with WS85 indicate a larger emittance growth than those obtained with WS64. Moreover, for the short period of time over which measurements with both instruments are available, a sort of systematic shift between them is observed. Based on the available data it can therefore only be concluded that the maximum emittance growth in the PS does not exceed $\approx 20 \%$ (as measured with WS85), and could actually be even smaller as indicated by WS64. Furthermore, the disagreement between the devices appears to increase with beam intensity. These discrepancies are expected to be the result of a wrong calibration of the WS85. The device was therefore exchanged during the 2017/18 winter technical stop to improve the accuracy of the measurement during the 2018 run. Nevertheless, measurements of the vertical emittance during the high-intensity period had to be performed with WS85, as WS64 occasionally delivered nonphysical results. 

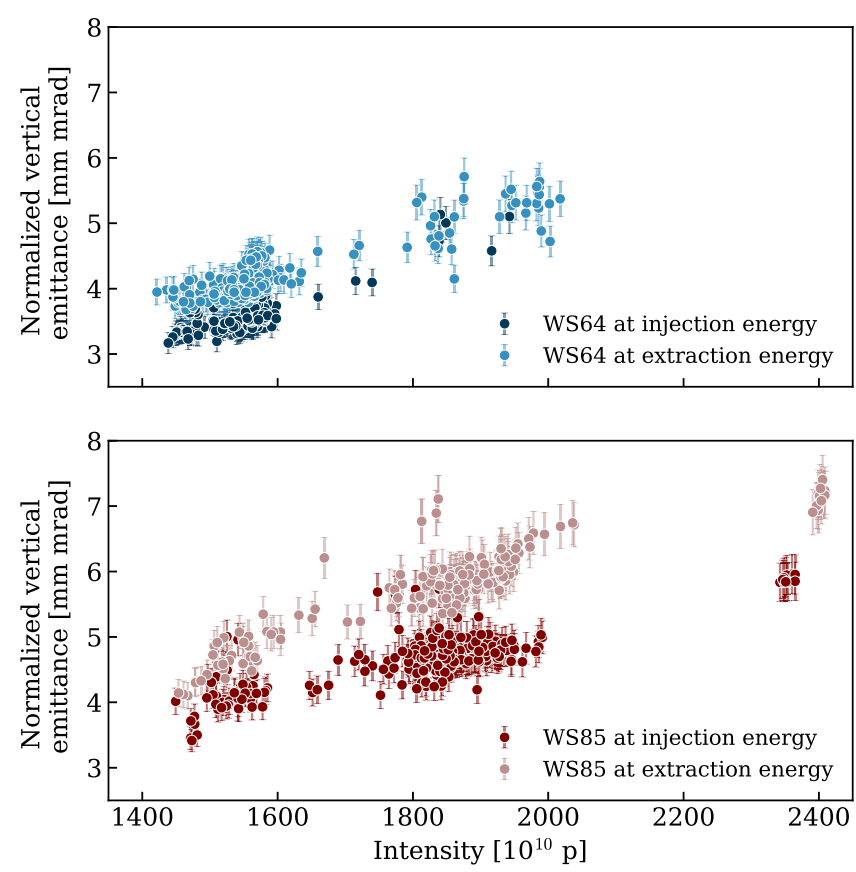

FIG. 8. Evolution of the vertical beam emittance versus intensity for the data shown in Fig. 7.

The same emittance data are also shown in Fig. 8 where the vertical emittance is plotted as a function of the beam intensity, distinguishing the data by measurement time along the PS cycle and WS used for the measurements.

The linear dependence of emittance as a function of total beam intensity is a consequence of the way the beam is produced at the PSB, namely with constant brightness $[16,17]$. The values of the parameters of the straight lines fitted to the data are shown in Table I. The behavior of the two instruments is different, as seen already in Fig. 7. WS64 features a slope almost independent on the energy of the measurements, while the constant term is increasing, which indicates a blow-up between injection and extraction energy. WS85 features a slope that depends sizeably on the energy, whereas the constant term is decreasing with

TABLE I. Parameters of the straight line $\epsilon_{y}\left(N_{\mathrm{p}}\right)=a N_{\mathrm{p}}+b$, where $\epsilon_{y}$ is the vertical emittance of the beam and $N_{\mathrm{p}}$ the total intensity, best fitting the data plotted in Fig. 8. Results for the injection and extraction energy are shown. For data measured with WS85 two cases are considered, namely either including or not the high-intensity data.

\begin{tabular}{lcrrrr}
\hline \hline & \multicolumn{2}{c}{ Injection energy } & & \multicolumn{2}{c}{ Extraction energy } \\
\cline { 2 - 3 } & $a\left[10^{-4}\right]$ & $\mathrm{B}$ & & $A\left[10^{-4}\right]$ & \multicolumn{1}{c}{$\mathrm{B}$} \\
\hline WS64 & $35 \pm 2$ & $-1.9 \pm 0.3$ & & $28 \pm 1$ & $-0.4 \pm 0.2$ \\
WS85 & $19 \pm 1$ & $1.1 \pm 0.2$ & $33 \pm 1$ & $-0.3 \pm 0.2$ \\
WS85 and & $21 \pm 1$ & $0.9 \pm 0.1$ & $31 \pm 1$ & $0.0 \pm 0.1$ \\
High-intensity & & & & \\
Data & & & & \\
\hline \hline
\end{tabular}

energy. In addition to blow-up along the cycle, this observation could be related to the already mentioned wrong calibration of the device. As expected, the addition of the high-intensity data does not significantly change the fit results.

The variation of the vertical beam emittance along the PS cycle has also been measured, and the results are shown in Fig. 9. The information about the beam intensity for each measured data point is also reported, to provide additional information on the possible correlation of the emittance fluctuations with the corresponding typical intensity variations. Indeed, dark-blue colors tend to be on the lower end of the emittance values, while dark-red colors are rather on the higher end of the emittance values, even though the correlation is not always respected.

Overall, the typical value of the emittance growth is about $20 \%$. A first increase in emittance occurs at injection, while after transition crossing, corresponding to $427 \mathrm{~ms}$ in the plot, an approximately linear growth is visible. Such an emittance growth is responsible for the change of fit parameters of the straight line as reported in Table I. Part of the emittance growth observed during the PS cycle could be possibly mitigated by properly tuning the machine working point. However, while this is relatively straightforward in the low-energy part of the cycle, after transition crossing the pole-face-windings circuits should be used to control transverse tunes and chromaticities and their use is by far nontrivial $[18,19]$.

During the measurement campaign performed to assess the MTE performance as a function of the total beam intensity, the extraction bumps (slow and fast) and the final value of the horizontal tune were reoptimized. This last point is important to be addressed as indirect space charge effects generate a shift of the transverse position of the beamlets at extraction [12]. This phenomenon was investigated in and confirmed by numerical simulations [13].

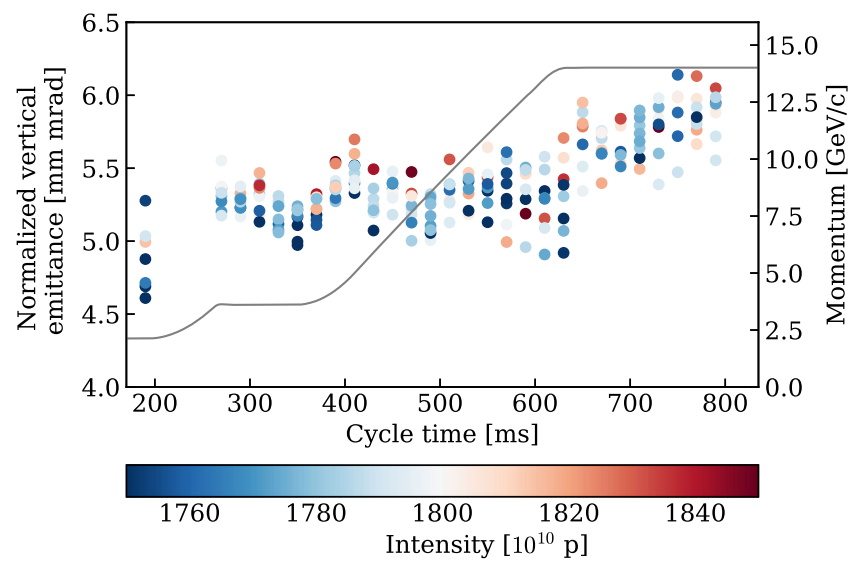

FIG. 9. Evolution of the vertical beam emittance, measured with WS85, along the PS MTE cycle. The color scale represents the typical intensity fluctuation occurring during the measurement. 

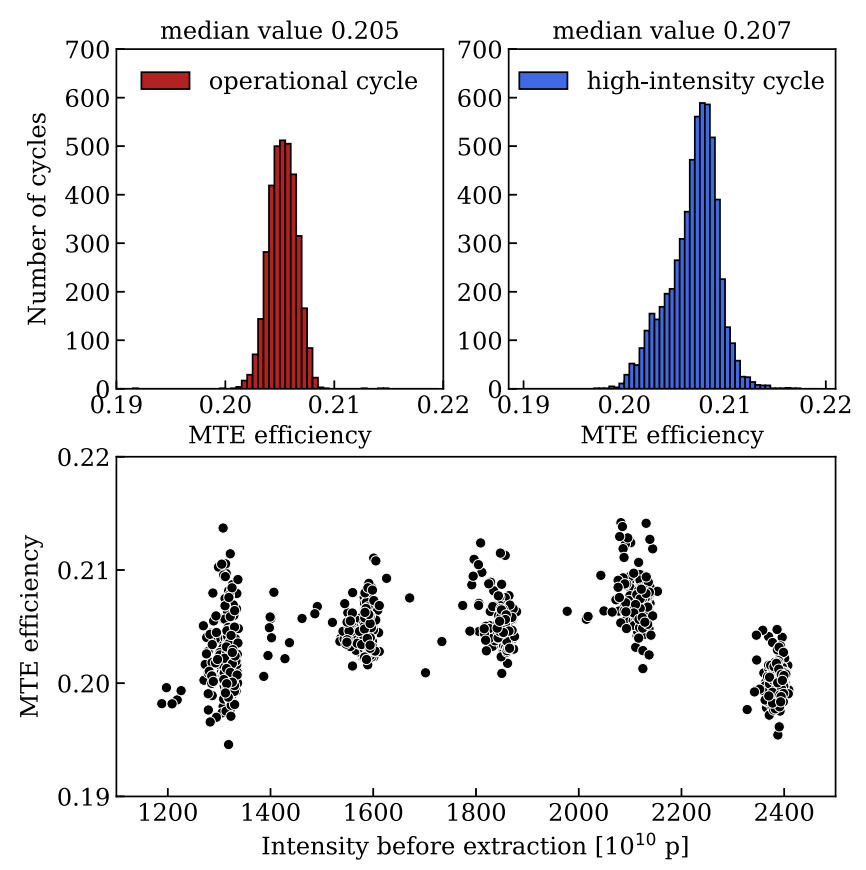

FIG. 10. Upper: Distribution of MTE efficiency for the operational (left) and the high-intensity variant (right). The highintensity data refer to the three-day period on November 11 , when the MTE beam with an intensity of about $2.4 \times 10^{13}$ protons was delivered to the SPS for the fixed-target physics (see also Fig. 7 , bottom). The median of the distribution is essentially the same, while a low-efficiency tail is present for the high-intensity beam. Lower: MTE efficiency for various beam intensities. These data refer to a special test that took place on November 15 for about 9 hours, without performing any beam optimization at each intensity step.

A comparison of the distribution of $\eta_{\mathrm{MTE}}$ for the operational beam in 2017 (typical intensity around 1.5 $1.6 \times 10^{13}$ protons) and for the beam specially prepared and optimized for SPS fixed-target physics with high-intensity MTE beam (typical intensity around $2.4 \times 10^{13}$ protons) is shown in Fig. 10 (upper). The high-intensity data refer to the three-day period on November 11 (see also Fig. 7, bottom). The two distributions are almost the same, featuring a similar median. The only difference is a larger tail skewed toward low values of $\eta_{\mathrm{MTE}}$ for the case of the high-intensity beam. This is not considered to be a fundamental issue as it could be fixed by working on the reproducibility of the intensity delivered by the Linac and the PSB.

In fact, as it can be seen in Fig. 10 (lower), while $\eta_{\text {MTE }}$ is practically constant for a wide range of beam intensities (essentially from the operational one up to about $2.2 \times 10^{13}$ protons), a small reduction is observed for the case of $2.4 \times 10^{13}$ protons. Hence, a fluctuating beam intensity could explain the tail. Note that these data refer to a special test that took place on November 15 for about 9 hours, without performing any beam optimization at each intensity step.
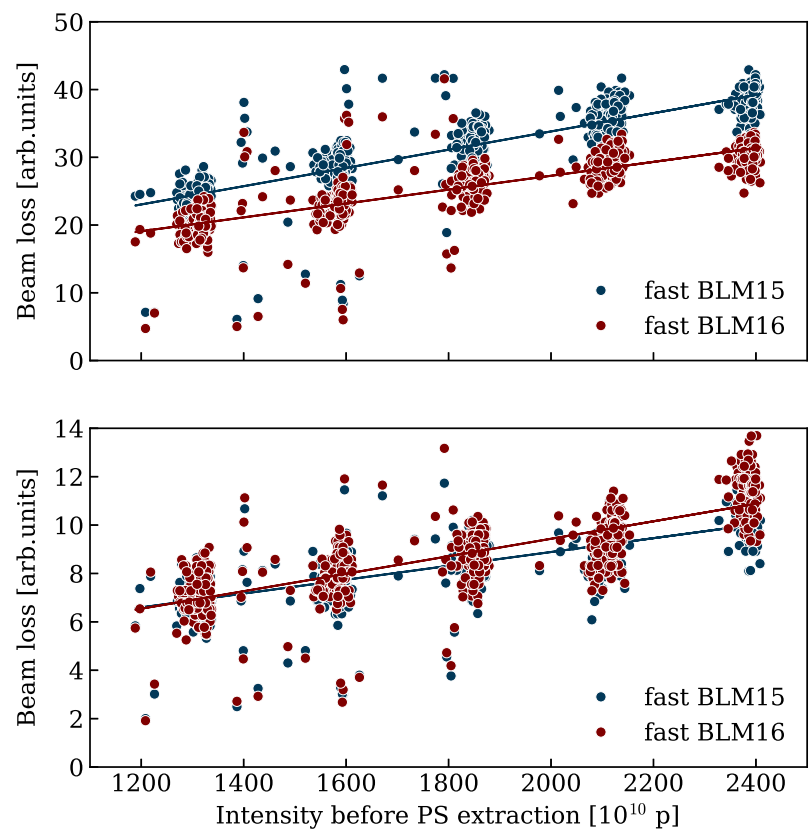

FIG. 11. Measured extraction beam losses for the islands (upper) and core (lower) as a function of the total beam intensity. The losses are measured in the extraction region, i.e., at the location of the dummy septum in SS15 and of the magnetic septum in SS16. The straight lines best fitting the data are also shown.

The third aspect considered during the tests was the evolution of the beam losses at extraction, which is also a means to evaluate whether the transverse beam properties are changing with intensity. Figure 11 reports the losses at extraction as measured by fast BLMs, which are devices capable of providing the turn-by-turn losses with subturn sampling rate. Thus, they allow to distinguish between the losses for the islands and the core.

The losses are shown as a function of intensity and are given for two key locations in the PS ring, namely the location of the so-called dummy septum [4-6] in straight section (SS) 15 and that of the magnetic extraction septum in SS16. It is worth mentioning that the lower losses for the extraction of the beam core are due to the faster rise time of the kickers, with respect to those used for the four islands. The important feature visible in the plots is that the increase of beam losses is to a large extent linear with intensity, thus indicating that no new phenomenon is appearing when the intensity is increased. The parameters of the straight lines best fitting the data are reported in Table II.

An example of the raw signal recorded with BLM15 is shown in Fig. 12. Additional useful information can be extracted from the analysis of the time evolution of the beam losses as measured by the fast BLMs in SS15 and 16, namely the dependence of the position of the peak of the losses on the total beam intensity. For this purpose, the rising edge of each loss peak is fitted with a Gaussian function, whose mean value and sigma are then consider for 
TABLE II. Parameters of the straight lines $\operatorname{BLM}\left(N_{\mathrm{p}}\right)=$ $a N_{\mathrm{p}}+b$, where $N_{\mathrm{b}}$ is the total intensity, best fitting the data plotted in Fig. 11. Results for the core and the islands are shown.

\begin{tabular}{lccccc}
\hline \hline & \multicolumn{2}{c}{ Core } & & \multicolumn{2}{c}{ Islands } \\
\cline { 2 - 3 } \cline { 5 - 6 } & $a\left[10^{-3}\right]$ & $\mathrm{B}$ & & $a\left[10^{-2}\right]$ & $\mathrm{B}$ \\
\hline BLM 15 & $2.86 \pm 0.07$ & $3.2 \pm 0.1$ & & $1.35 \pm 0.02$ & $6.9 \pm 0.4$ \\
BLM 16 & $3.61 \pm 0.08$ & $2.2 \pm 0.2$ & & $1.02 \pm 0.02$ & $6.8 \pm 0.4$ \\
\hline \hline
\end{tabular}

the subsequent analysis. The falling edge of the loss peak is discarded, as its shape is determined by the characteristics of the electronics.

The dependence of this mean position on the beam intensity is shown in Fig. 13 for the island (upper) and the core (bottom) for both locations of the fast BLMs. While a linear shift with intensity of the time of the peak losses is clearly observed for the island, no shift is visible for the core. This effect is linked with the transverse displacement of the fixed-point positions as a function of intensity as reported in [12], which was understood to be caused by indirect space charge effects as discussed in [13]. The experimental observations are in agreement with expectations: the distance of the fixed-point position from the origin increases proportionally to the beam intensity and the position of the loss peak therefore decreases with intensity, as particles at larger amplitude will be intercepted earlier by the septum. These results reveal that the fast
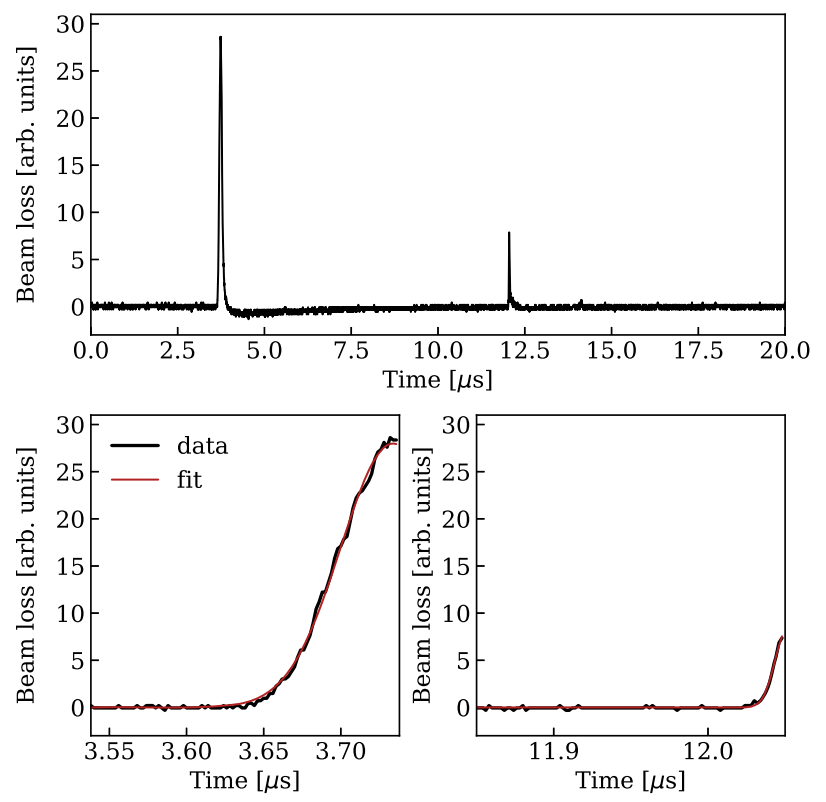

FIG. 12. Example of a fast BLM signal recorded in SS15 (top). The losses generated by the island sweeping through the blade of the dummy septum cause the first spike, while the second one represents the losses of the core. The bottom plots present detailed views of the island and core losses (left and right plot, respectively) together with the fitted Gaussian model.
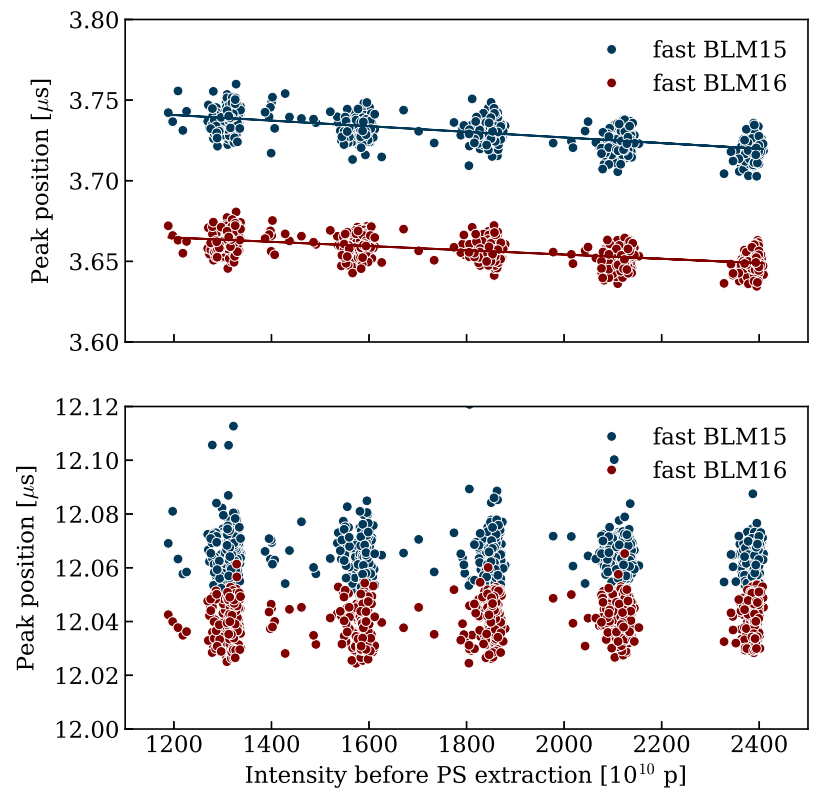

FIG. 13. Measured time of the peak losses for the island (upper) and the core (bottom) at the locations of the fast BLMs in SS15 and SS16. The linear shift is visible only for the island.

BLMs can be used to extract much more information than only beam losses, and this will be further elaborated on in the next section.

\section{B. Digression: Extracting optics- and beam-related parameters from fast BLM signals}

In this section a more involved treatment of the data from the fast BLMs is proposed and some additional results are presented.

The horizontal beam distribution can be described by a Gaussian according to

$$
\rho(x, t)=\frac{N_{\mathrm{p}}}{\sqrt{2 \pi} \sigma} \exp \left[-\frac{1}{2}\left(\frac{x-\mu(t)}{\sigma}\right)^{2}\right],
$$

where $N_{\mathrm{p}}$ is the intensity, $\sigma$ the size of the beam, and $\mu(t)$ represents the displacement during the rise time of the kickers. $\mu(t)$ can be described as

$$
\mu(t)=\frac{A}{2}\left\{\sin \left[2\left(t-\frac{\pi}{4} \zeta\right) \frac{1}{\zeta}\right]+1\right\}
$$

where $A$ is the maximum amplitude of the horizontal beam displacement at the location where the losses are measured and

$$
\zeta=\frac{\tau_{\alpha}}{\arcsin (1-2 \alpha)}
$$

where $\tau_{\alpha}$ stands for the time during which the kicker sweeps particles through the amplitude interval 
$[\alpha A,(1-\alpha) A]$. Typically, we use $\alpha=0.1$ and $\tau_{0.1}=$ $350 \mathrm{~ns}$ and $80 \mathrm{~ns}$ for the kickers extracting the islands and the core, respectively [2]. The measured losses $\ell(t)$ can be obtained as

$$
\begin{aligned}
\ell(t)= & \mathcal{F}(t) \times \frac{N_{\mathrm{p}}}{\sqrt{2 \pi} \sigma} \frac{\tau_{\alpha}}{\tau_{\text {rev }}} \\
& \times \int_{x_{\mathrm{s}}-\Delta_{\mathrm{s}} / 2}^{x_{\mathrm{s}}+\Delta_{\mathrm{s}} / 2} \exp \left[-\frac{1}{2}\left(\frac{x-\mu(t)}{\sigma}\right)^{2}\right] d x
\end{aligned}
$$

where $x_{\mathrm{s}}$ and $\Delta_{\mathrm{s}}$ are the central position and width of the dummy septum (or magnetic septum) blade, respectively, and $\tau_{\text {rev }}$ represents the revolution time, i.e., $2.1 \mu \mathrm{s}$ at $14 \mathrm{GeV} / \mathrm{c}$. Equation (6) expresses the losses as the fraction of the horizontal beam distribution intercepting the blade of the septum (dummy or magnetic) times the typical response of the BLM to the proton losses represented by the calibration function $\mathcal{F}(t)$. The time dependence has been included into $\mathcal{F}(t)$ to account for effects such as the change of the beam angle at the location of the septum during the rise time of the kickers. Such a calibration factor is currently unknown and a campaign of numerical simulations is planned to determine it and its dependence on the beam angle.

Note also that $x_{\mathrm{s}}=\hat{x}_{\mathrm{s}}-\hat{x}_{\mathrm{b}}-\hat{x}_{\mathrm{co}}$ holds true, where $\hat{x}_{\mathrm{s}}$, $\hat{x}_{\mathrm{b}}$, and $\hat{x}_{\mathrm{co}}$ represent the dummy septum position with respect to the zero closed orbit, the amplitude of the slow bump, and the value of the closed orbit, respectively. Of course, these quantities depend on the location along the ring and on whether the island or the core are considered.

From Eq. (5) it is possible to evaluate $\sigma$ from the knowledge of $\ell(t)$ (obtained from beam measurements), $\mathcal{F}(t)$ (obtained from numerical simulations), and $\mu(t)$ (obtained from the characteristics of the kicker magnets and of the ring optics).

This approach can be used to determine emittance and momentum spread of the core and island, the former being a fundamental parameter for the beam quality, by combining the data from the BLMs in SS15 and 16. Knowledge of the optical functions of core and island and of the calibration factor $\mathcal{F}(t)$, with all this information coming from simulation studies, shall allow to extract these quantities from the signals of the fast BLMs in SS15 and 16. Nevertheless, it is worthwhile stressing that the calibration factor $\mathcal{F}(t)$ is still to be determined and, therefore, such a measurement is currently not possible. Eventually, the described procedure could become a new means to monitor the beam quality. In the meantime, monitoring the width of the BLM signals should provide a means to indirectly detect variations of the beam parameters and hence react accordingly.

It is also worth mentioning that in case the emittance and the momentum spread would be provided by an independent measurement, e.g., performed at the SPS, the previous discussion can be adapted to infer the optical parameters from the beam sigma obtained from the BLM signals.

\section{SPS results}

The final step of the high-intensity studies was the delivery of the optimized beam from the PS to the SPS. The goal of the studies reported in this section was the comparison of the performance of the high-intensity to the operational MTE beam in terms of transmission through various stages of the SPS cycle. No particular attention was payed to the transmission through the transfer line joining the PS and the SPS, as it was found to be essentially independent of the total beam intensity.

The SPS performance is based on the definition of key times along the magnetic cycle at which the transmission efficiencies are evaluated. The classification of the different stages and their times within the cycle are defined in the following way: (i) Injection 1 and 2, referring to the injection efficiency of each individual PS pulse, (ii) Flat bottom, referring to the moment $40 \mathrm{~ms}$ after Injection 2, (iii) Front porch, referring to $200 \mathrm{~ms}$ after flat bottom, and (iv) Transition, referring to $70 \mathrm{~ms}$ after front porch.

These times can also be seen in Fig. 14, where the evolution of the beam intensity and momentum is shown as a function of time. Note that transmission efficiencies (ii)-(iv) are defined in terms of the transmission from the previous stage to the stage named. As an example, the transmission at transition is defined as the percentage of transmitted intensity from front porch to transition.

The main outcome of these tests, as far as the SPS ring performance is concerned, is reported in Fig. 15, where the transmission through the various stages of the SPS cycle is shown. In the upper part of the figure, the magnetic cycle of the SPS is shown together with the evolution of the beam intensity for both the operational and the high-intensity case. During the high-intensity tests the beam was systematically dumped during the energy ramp, after crossing the transition energy, to prevent too high mechanical stresses on the dump in case of disposal of the beam at top energy. In the lower part of the figure, the analysis of

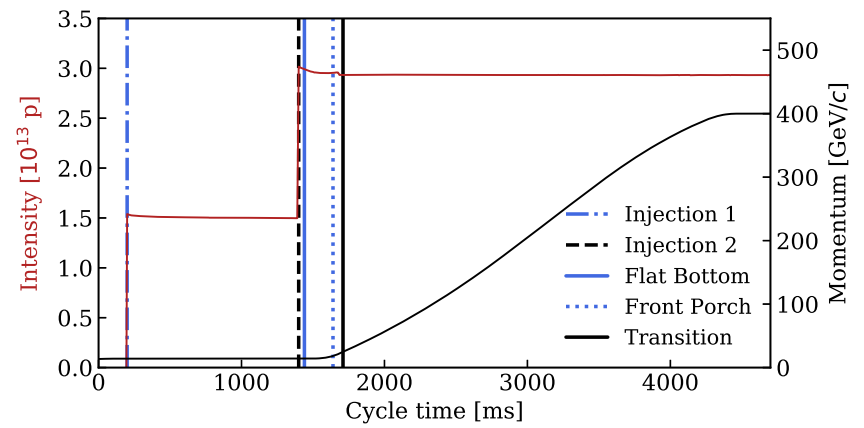

FIG. 14. Example of beam intensity in the SPS as a function of time. The vertical lines indicate the different stages at which the transmission efficiencies are evaluated. 

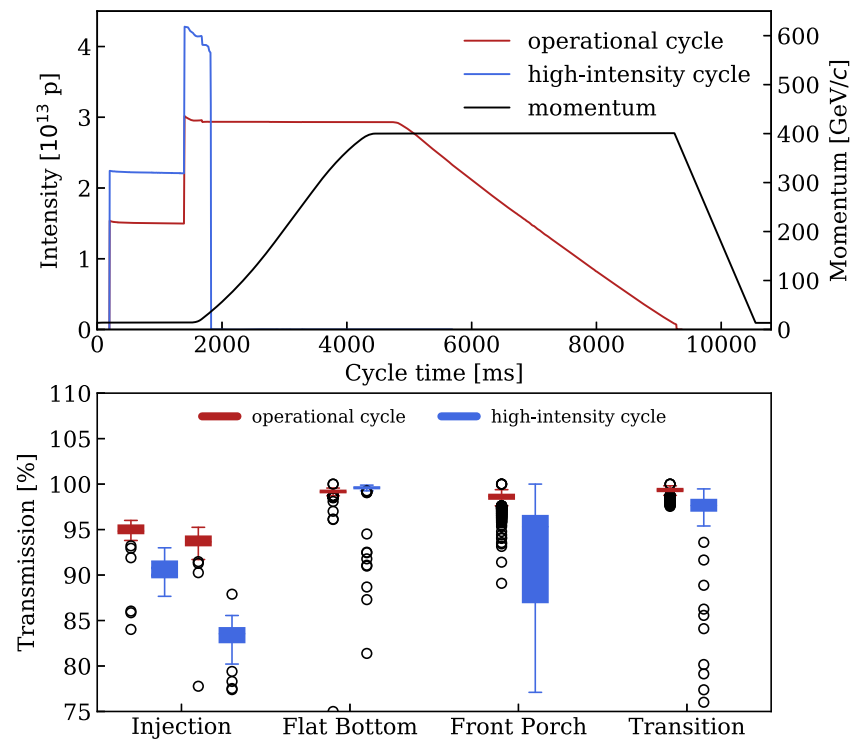

FIG. 15. Upper: SPS magnetic cycle and beam intensity for the operational and the high-intensity cycle. For the case of the highintensity cycle, the beam is dumped on the energy ramp, which explains the sudden total beam loss. Lower: Beam transmission in the SPS between the various stages of its cycle. The performance at injection is separated into first and second batch injected from the PS. For the remaining three stages, only data where both injections were present were considered. The open circles indicate outliers in the distributions of transmission efficiency.

the transmission is reported. The performance of both injected batches from the PS is reported, revealing a significant reduction in transmission for the second batch. Given the allocated time for these tests, this result does not come as a surprise, as the main effort was devoted to optimizing the first injection as a proof of principle. In future studies the proper optimisation of the second injection will be further addressed.

In comparison to the operational MTE beam, a reduction of transmission efficiency at injection is visible for the highintensity beam. This is mainly due to the large vertical emittance, which goes beyond the vertical SPS acceptance, and therefore dominates the performance at injection. Looking at Fig. 15 one finds that the beam losses at injection amount to $\approx 5 \%$ and $\approx 9 \%$ for the nominal and the highintensity beam, respectively. In order to improve the accuracy of these values, their measurement is based on the ring transformers in both the PS and the SPS rather than the single passage transformers in the transfer line. The transmission of the first injection is therefore calculated using the last and the first measurement point in the PS and the SPS, respectively. For the second injection the same approach was taken in the PS, whereas the difference between circulating intensity and intensity measured immediately after the second injection was calculated from the SPS data.

The PS extraction losses can be estimated to $\approx 2 \%$. The typical vertical emittance values for the MTE beams are

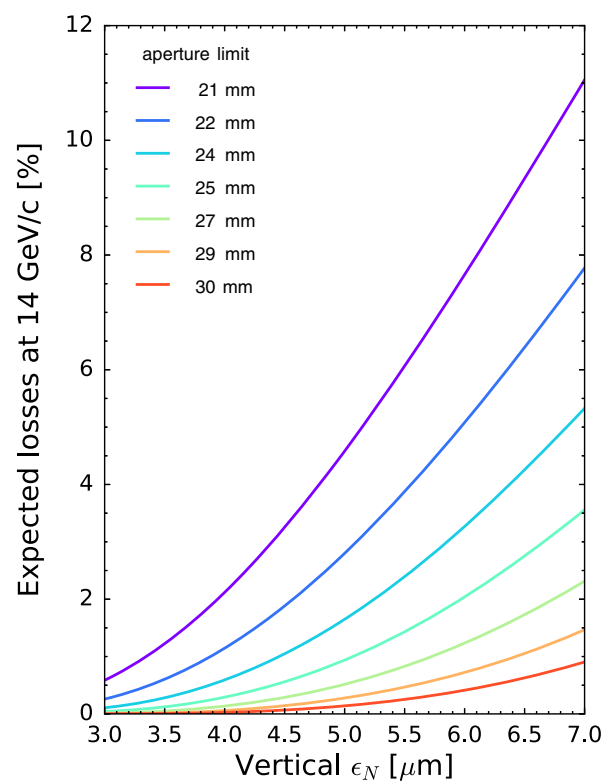

FIG. 16. Expected losses at the SPS flat bottom as a function of the vertical normalized emittance due to the global aperture bottleneck (transition between the MBA and MBB dipoles). Several cases are shown, depending on the estimated available aperture. The aperture at the time of the high-intensity MTE beam measurements is expected to be $30 \mathrm{~mm}$ (adapted from [5]).

$\approx 5 \mu \mathrm{m}$ and $\approx 7 \mu \mathrm{m}$ for the nominal- and high-intensity beam, respectively. At the time of the MTE tests, the bottleneck of the SPS aperture was located at the transition between the MBA and MBB dipoles and measured to be $\approx 30 \mathrm{~mm}[20,21]$. Based on Fig. 16, where the expected dependence of beam loss on the vertical emittance is shown, the beam loss due to the SPS ring aperture should be $\approx 0 \%$ and $\approx 1 \%$ for the nominal and high-intensity beam, respectively. Subtracting the PS extraction losses and these losses at the SPS aperture from the values obtained from Fig. 15, it can be concluded that $\approx 3 \%$ and $\approx 6 \%$ of the losses can be attributed to an additional aperture limitation located directly at injection into the SPS.

To validate this consideration one can assume a Gaussian beam distribution with emittance $\epsilon$ in the vertical plane, and the relative beam losses due to an aperture of normalized value $a$ are then given by

$$
\ell(a, \epsilon)=\frac{2}{\sqrt{2 \pi \epsilon}} \int_{a}^{\infty} \exp \left[-\frac{1}{2}\left(\frac{x}{\sqrt{\epsilon}}\right)^{2}\right] d x
$$

If $\epsilon_{\mathrm{op}}$ and $\epsilon_{\mathrm{hi}}$ stand for the value of the normalized emittance of the operational and high-intensity beams, respectively $\left(\epsilon_{\mathrm{hi}} \geq \epsilon_{\mathrm{op}}\right)$, it is easy to show that the following holds

$$
\ell\left(a, \epsilon_{\mathrm{hi}}\right)=\ell\left(a \sqrt{\frac{\epsilon_{\mathrm{op}}}{\epsilon_{\mathrm{hi}}}}, \epsilon_{\mathrm{op}}\right) .
$$


Let us indicate the aperture limitation at injection as $a_{\mathrm{inj}}$, for which $\ell\left(a_{\mathrm{inj}}, \epsilon_{\mathrm{op}}\right)=0.03$ holds true. It is then clear that the following holds

$$
\begin{aligned}
& \ell\left(a_{\mathrm{inj}}, \epsilon_{\mathrm{hi}}\right)-\ell\left(a_{\mathrm{inj}}, \epsilon_{\mathrm{op}}\right) \\
& \quad=\frac{2}{\sqrt{2 \pi \epsilon_{\mathrm{op}}}} \times \int_{\phi a_{\mathrm{inj}}}^{a_{\mathrm{inj}}} \exp \left[-\frac{1}{2}\left(\frac{x}{\sqrt{\epsilon_{\mathrm{op}}}}\right)^{2}\right] d x, \\
& \phi=\sqrt{\frac{\epsilon_{\mathrm{op}}}{\epsilon_{\mathrm{hi}}}} .
\end{aligned}
$$

This expresses a relationship between the losses at injection for the two types of beams assuming the same aperture limit and different emittances. Evaluation of Eq. (8) provides an estimate of the loss difference of $\approx 2.8 \%$ to be compared with the measured value of $\approx 3 \%$, which is in rather good agreement.

Another observation supporting the claim that the losses at injection are mainly driven by vertical emittance-related phenomena is that in all further stages of the SPS cycle the high-intensity beam performs similarly to the operational one. At least, one can state that the high-end part of the distribution of transmission values is comparable with that of the operational beam. It should be noted that the start of acceleration and transition crossing, which require careful adjustment of the machine parameters, could not be optimized completely during the time allocated for the tests. The large spread in the value of the transmission is due to the fact that all data, even during the setting up process, are included in the analysis shown. The main observation is that the maximum of the wide distribution of transmission of the high-intensity beam is comparable to the narrow distribution of transmission of the operational beam.

These results were considered as confirmation that the SPS can perform equally well with both operational and high-intensity beams as long as sufficient commissioning time is allocated and a smaller vertical beam emittance is provided by the PS. Reduced emittances will eventually become available as the improvements planned in the framework of the LHC Injectors Upgrade (LIU) project at CERN [22] are being implemented during the currently ongoing Long Shutdown 2 (LS2).

\section{CONCLUSIONS}

MTE started operation in the second half of 2015, replacing the CT extraction mode. Since then, the MTE performance has been constantly improved, in particular in the SPS, thus approaching that of CT.

A high-intensity version of MTE was produced in 2017 and tests were carried out in the PSB, the PS, and the SPS. For the first time a systematic study of the performance of the highly-sophisticated MTE beam under the influence of strong intensity-dependent effects was carried out. At the
PSB the main challenge was the generation of the highintensity beams with low extraction losses while fulfilling the constraints imposed on the transverse emittances by either the PS, i.e., a large horizontal emittance to optimize the MTE efficiency, or the SPS, i.e., a small vertical emittance to minimize the injection losses due to the vertical acceptance. This was successfully achieved, thus allowing us to move to the downstream machines.

In the PS, the performance of high-intensity MTE beams is comparable with that of the operational beam. The SPS performance is dominated by the value of the vertical emittance delivered by the PS, where the emittance delivered by the PSB is to a large extent preserved. The larger vertical emittance with respect to the operational beam explains the larger losses observed and the experimental data agree well with the data known about the SPS acceptance, as it was shown in the paper. Considering this aspect, it is clear that the novel LIU beams will mitigate this limitation. All in all, in spite of the very limited set-up time, no show-stopper to further improve the overall performance was found.

Furthermore, the experimental campaign showed the usefulness of the fast BLMs installed in the extraction region, well beyond the original goal of measuring the beam losses for island and core. Indeed, it was possible to assess that the island's position is changing as a function of beam intensity, while that of the core is insensitive to it. More than that, we outlined a method to derive the beam size from loss measurements, for which the calibration factor of the BLMs needs to be determined. Studies will be launched to fill this gap, in view of quantitatively assessing the proposed approach and applying it in operation.

Based on the successful outcome of the tests carried out in 2017, it was formally decided to discontinue CT operation and to dismantle the corresponding hardware during LS2 so that all future SPS fixed-target physics runs will be based on MTE, no matter the value of the requested beam intensity. This is an excellent outcome of the intense studies and it is of paramount importance as it confirms that MTE has reached full maturity to become the reference for the SPS fixed-target physics programme at CERN.

It is also worth mentioning that a parallel research activity aims at implementing a barrier bucket rf manipulation in the PS ring. In this way, a gap in the longitudinal structure of the beam can be created. In case of positive outcome of these studies, such gymnastics could be applied prior to extraction of the MTE beams, with the extraction kickers fired synchronously with the gap created by the barrier bucket. Such a scheme would allow us to further reduce extraction losses and would pave the way to produce even more intense proton beams [23].

\section{ACKNOWLEDGMENTS}

We would like to express our warm thanks to the operation crews of the PSB, the PS, and the SPS 
synchrotrons, who provided invaluable support during the experimental studies presented and discussed in this paper. Furthermore, we would like to express our gratitude to the anonymous referees for the excellent comments made on the original version of the manuscript.

\section{APPENDIX: MAIN PARAMETERS OF THE PS AND SPS RINGS}

In this Appendix the main parameters of the PS and SPS rings are reported for the sake of completeness.

TABLE III. Basic parameters of the PS ring. The cycle length refers to the time needed from injection up to extraction energy (including the time for single-, multiturn, or slow beam extraction) and back again to injection. The cycle length is a multiple of the PSB repetition rate, which is $1.2 \mathrm{~s}$.

\begin{tabular}{ll}
\hline \hline Accelerated particles & $\mathrm{p}$ and several types of ions \\
Injection kinetic energy [GeV] & 1.4 \\
Maximum energy [GeV] & 26 \\
Circumference [m] & $200 \pi$ \\
Magnetic lattice & Alternating-gradient, \\
Focusing & Combined-function \\
Focusing type & FOFDOD \\
Number of main magnets & 100 \\
Bending magnetic field [T] & 0.101 at injection, \\
& 1.24 at $26 \mathrm{GeV}$ \\
Betatron oscillations/turn & 6.25 (h), 6.25 (v) \\
Minimum and maximum & $11.7-22.6$ \\
$\beta$ function [m] & \\
Minimum and maximum & $2.3-3.1$ \\
Dispersion function [m] & 5.72 \\
Transition energy [GeV] & 1.2 (up to $20 \mathrm{GeV})$ \\
Magnetic cycle length [s] & 2.4 (up to $26 \mathrm{GeV})$ \\
& number $=100$, \\
Straight sections & 80 of $1.6 \mathrm{~m}, 20$ of $3 \mathrm{~m}$ \\
Main rf system (tunable) & $10+1$ cavities, \\
& $2.6-9.5 \mathrm{MHz}$, \\
Auxiliary rf systems [MHz] & $200 \mathrm{kV}$ total maximum \\
\hline \hline
\end{tabular}

TABLE IV. Basic parameters of the SPS ring. The cycle length refers to the time needed from injection up to extraction energy (including the time for single-turn or slow beam extraction) and back again to injection. The cycle length is a multiple of the PSB repetition rate, which is $1.2 \mathrm{~s}$.

\begin{tabular}{ll}
\hline Accelerated particles & $\mathrm{p}$ and several types of ions \\
Injection energy [GeV] & 14 \\
Maximum energy [GeV] & 450 \\
Circumference [m] & $2200 \pi$ \\
Magnetic lattice & Alternating-gradient, \\
Focusing & Separated-function \\
Focusing type & FODO \\
Number of main magnets & 744 dipoles, 216 quadrupoles \\
Bending magnetic field [T] & 0.06 at injection, \\
& 2.03 at $450 \mathrm{GeV}$ \\
Betatron oscillations/turn & 26.62 (h), 26.58 (v) \\
Minimum and maximum & \\
$\beta$ function [m] & $20-107$ \\
Minimum and maximum & \\
Dispersion function [m] & $-0.5-4.5$ \\
Transition energy [GeV] & 21.6 \\
Magnetic cycle length [s] & 10.8 (up to $400 \mathrm{GeV})$ \\
Long straight & number $=6$, \\
Sections & $130 \mathrm{~m}$ each \\
Main rf system & 4 cavities, \\
& $200 \mathrm{MHz}$, \\
Auxiliary rf systems [MHz] & $7 \mathrm{MV}$ total maximum \\
\hline \hline
\end{tabular}

[1] R. Cappi and M. Giovannozzi, Novel Method for Multiturn Extraction: Trapping Charged Particles in Islands of Phase Space, Phys. Rev. Lett. 88, 104801 (2002).

[2] M. J. Barnes et al., The CERN PS multi-turn extraction based on beam splitting in stable islands of transverse phase space: Design, CERN Report No. CERN-2006-011, 2006.

[3] E. Benedetto et al., Results from the 2009 Beam Commissioning of the CERN Multi-turn Extraction, in First International Particle Accelerator Conference, edited by A. Noda, C. Petit-Jean-Genaz, V. R. W. Schaa, T. Shirai, and A. Shirakawa (JACoW, Geneva, 2010), p. 3619.

[4] J. Borburgh, S. Damjanovic, S. Gilardoni, M. Giovannozzi, C. Hernalsteens, M. Hourican, A. Huschauer, K. Kahle, G. Le Godec, O. Michels, and G. Sterbini, First implementation of transversely split proton beams in the CERN Proton
Synchrotron for the fixed-target physics programme, Europhys. Lett. 113, 34001 (2016).

[5] S. Abernethy et al., Operational performance of the CERN injector complex with transversely split beams, Phys. Rev. Accel. Beams 20, 014001 (2017).

[6] A. Huschauer, A. Blas, J. Borburgh, S. Damjanovic, S. Gilardoni, M. Giovannozzi, M. Hourican, K. Kahle, G. Le Godec, O. Michels, G. Sterbini, and C. Hernalsteens, Transverse beam splitting made operational: key features of the multi-turn extraction at the CERN Proton Synchrotron, Phys. Rev. Accel. Beams 20, 061001 (2017).

[7] A. Huschauer, M. Giovannozzi, O. Michels, A. Nicoletti, and G. Sterbini, Analysis of performance fluctuations for the CERN Proton Synchrotron multi-turn extraction, J. Phys. Conf. Ser. 874, 012072 (2017). 
[8] C. Bovet, D. Fiander, L. Henny, A. Krusche, and G. Plass, The fast shaving ejection for beam transfer from the CPS to the CERN $300 \mathrm{GeV}$ machine, IEEE Trans. Nucl. Sci. 20, 438 (1973).

[9] J. Barranco García and S. Gilardoni, Simulation and optimisation of beam losses during continuous transfer extraction at the CERN Proton Synchrotron, Phys. Rev. Accel. Beams 14, 030101 (2011).

[10] K. Elsener et al., The CERN neutrino beam to Gran Sasso (NGS): conceptual technical design, CERN Report No. CERN-98-02, No. INFN-AE-98-05, 1998.

[11] G. De Lellis, Search for hidden particles (SHiP): A new experiment proposal, Nucl. Part. Phys. Proceedings 263-264, 71 (2015).

[12] S. Gilardoni, M. Giovannozzi, and C. Hernalsteens, First observations of intensity-dependent effects for transversely split beams during multiturn extraction studies at the CERN Proton Synchrotron, Phys. Rev. Accel. Beams 16, 051001 (2013).

[13] S. Machida, C. Prior, S. Gilardoni, M. Giovannozzi, A. Huschauer, and S. Hirlander, Numerical investigation of space charge effects on the positions of beamlets for transversely split beams, Phys. Rev. Accel. Beams 20, 121001 (2017).

[14] A. Huschauer, H. Bartosik, S. Cettour Cave, M. Coly, D. Cotte, H. Damerau, G. P. Di Giovanni, S. Gilardoni, M. Giovannozzi, V. Kain, E. Koukovini-Platia, B. Mikulec, G. Sterbini, and F. Tecker, Approaching the High-Intensity Frontier Using the Multi-Turn Extraction at the CERN Proton Synchrotron, in Proc. 61st ICFA ABDW HB2018, Daejeon, Korea, June 2018, edited by D. Jeon, D. Kim, and V. R. W. Schaa (JACoW, Geneva, 2018), pp. 231-236.

[15] C. Hernalsteens, T. Bach, S. Gilardoni, M. Giovannozzi, A. Lachaize, G. Sterbini, R. Tomás, and R. Wasef, CERN PS optical properties measured with turn-by-turn orbit data, in Proceedings of the 4th International Particle Accelerator Conference, IPAC-2013, Shanghai, China, 2013, edited by Z. Dai, C. Petit-Jean-Genaz, V. R. W. Schaa, and C. Zhang (JACoW, Shanghai, China, 2013), paper WEPEA054, pp. 2627-2629.

[16] V. Raginel, E. Benedetto, C. Carli, and B. Mikulec, Multi-Turn Injection of $50 \mathrm{MeV}$ Protons Into the CERN Proton Synchrotron Booster, in Proc. North American Particle Accelerator Conf. (NAPAC'13), Pasadena, CA, USA, Sep.-Oct. 2013, paper TUODB3, pp. 442-444, http://accelconf.web.cern.ch/AccelConf/PAC2013/papers/ tuodb3.pdf.
[17] E. Benedetto, M. Cieslak-Kowalska, V. Forte, and F. Schmidt, Space Charge Effects and Mitigation in the CERN PS Booster, in View of the Upgrade, in Proc. 57th ICFA ABDW HB2016, Malmö, Sweden, July 2016, edited by M. Eshraqi, G. Trahern, and V. R. W. Schaa (JACoW, Geneva, 2016), pp. 517-522.

[18] P. Freyermuth, D. Cotte, M. Delrieux, H. Genoud, S. Gilardoni, K. Hanke, O. Hans, S. Mataguez, G. Métral, F. Peters, R. Steerenberg, and B. Vandorpe, CERN proton synchrotron working point matrix for extended pole face winding powering scheme, in Proceedings of the International Particle Accelerator Conference, Kyoto, Japan, edited by A. Noda, Ch. Petit-Jean-Genaz, V. R. W. Schaa, T. Shirai, and A. Shirakawa (ICR, Kyoto, 2010), paper THPE019, pp. 4551-4553.

[19] A. Huschauer, Working point and resonance studies at the CERN Proton Synchrotron, Master's thesis, Vienna University of Technology, 2012, Report No. CERN-THESIS2012-212.

[20] V. Kain, H. Bartosik, S. Cettour Cave, K. Cornelis, and F. M. Velotti, Automatic local aperture measurements in the SPS, in Proc. 8th Int. Particle Accelerator Conf. (IPAC'17), Copenhagen, Denmark, May 2017 (JACoW, Geneva, 2017), pp. 4073-4076.

[21] V. Kain, R. Alemany-Fernandez, H. Bartosik, S. Cettour Cave, K. Cornelis, P. Cruikshank, J. Ferreira Somoza, B. Goddard, and C. Pasquino, Identification and removal of SPS aperture limitations, in Proc. 9th Int. Particle Accelerator Conf. (IPAC'18), Vancouver, Canada, Apr.-May 2018, pp. 709-712, http://accelconf.web.cern.ch/Accel Conf/ipac2018/papers/tupaf021.pdf.

[22] H. Damerau, A. Funken, R. Garoby, S. Gilardoni, B. Goddard, K. Hanke, A. Lombardi, D. Manglunki, M. Meddahi, B. Mikulec, G. Rumolo, E. Shaposhnikova, M. Vretenar, and J. Coupard, LHC Injectors Upgrade, Technical Design Report, Vol. I: Protons, Report No. CERN-ACC-2014-0337, 2014.

[23] M. Vadai, A. Alomainy, H. Damerau, S. S. Gilardoni, M. Giovannozzi, and A. Huschauer, Beam manipulations with barrier buckets in the CERN PS, in Proc. 10th Int. Particle Accelerator Conf. (IPAC'19), Melbourne, Australia, May 2019, edited by M. Boland, H. Tanaka, D. Button, R. Dowd, V.R. W. Schaa, and E. Tan (JACoW, Geneva, 2019), paper MOPTS107, pp. 1132-1135.

Correction: A misspelling in the Appendix heading was introduced during the production process and has been fixed. 